\title{
LATEX DYE CONFIRMATION OF LOCAL ANESTHETIC SPREAD IN PERICAPSULAR NERVE GROUP (PENG) BLOCK
}

\author{
J. Hall MD, S. Clendenen MD. \\ Mayo Clinic Florida, Anesthesiology \& Perioperative Medicine, Jacksonville FL, USA..
}

\section{Background}

Ultrasound-guided Pericapsular Nerve Group (PENG) block was recently described in the literature as a novel approach to provide regional anesthesia to the hip. Traditional blocks such as femoral and fascia iliaca frequently provide incomplete analgesia due to inconsistent coverage of proximal articular branches of the femoral(FN) and obturator nerves(ON). The articular branches from the FN to the hip joint enter the iliacus muscle at the L4-L5 level and course deep to the psoas muscle and tendon before innervating the hip capsule. The obturator nerve courses deep to the medial aspect of psoas muscle around the L5 level. The PENG block relies on cephalad spread of local anesthetic along the psoas muscle to cover these nerves more completely. We sought to confirm this with injection and direct visualization of dye in a cadaver.

\section{Results}

Dye was visualized along the fascial plane of the psoas interfacing with the articular branches of the $\mathrm{FN}$ and $\mathrm{ON}$ as seen below.

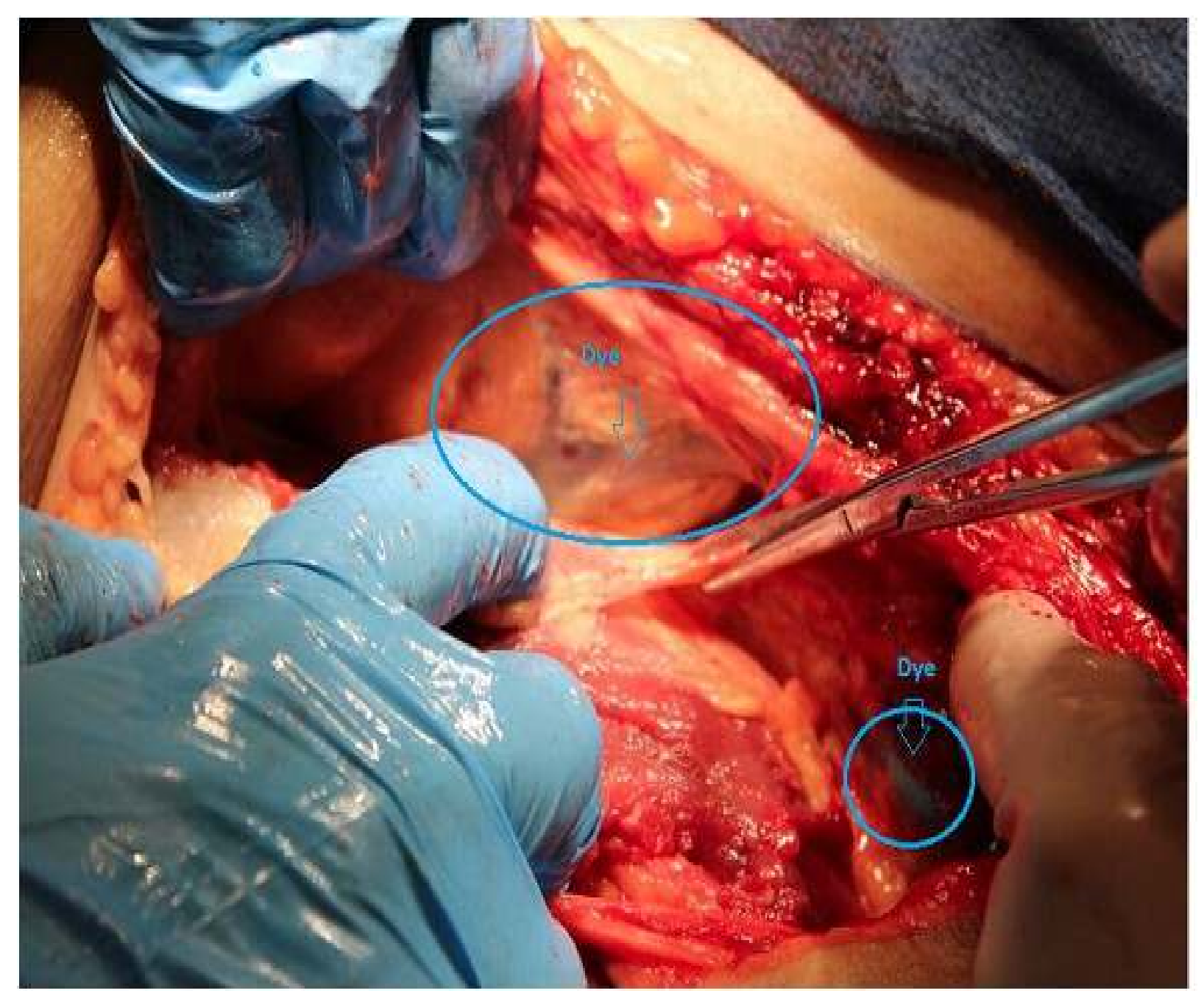

\section{Methods}

$20 \mathrm{cc}$ of blue latex dye was injected via an $18 \mathrm{~g}$ $10 \mathrm{~cm}$ needle into a male cadaver hip under ultrasound guidance using an in-plane method. The region was then dissected via an incision along the inguinal crease and exploring cephalad along the psoas muscle.
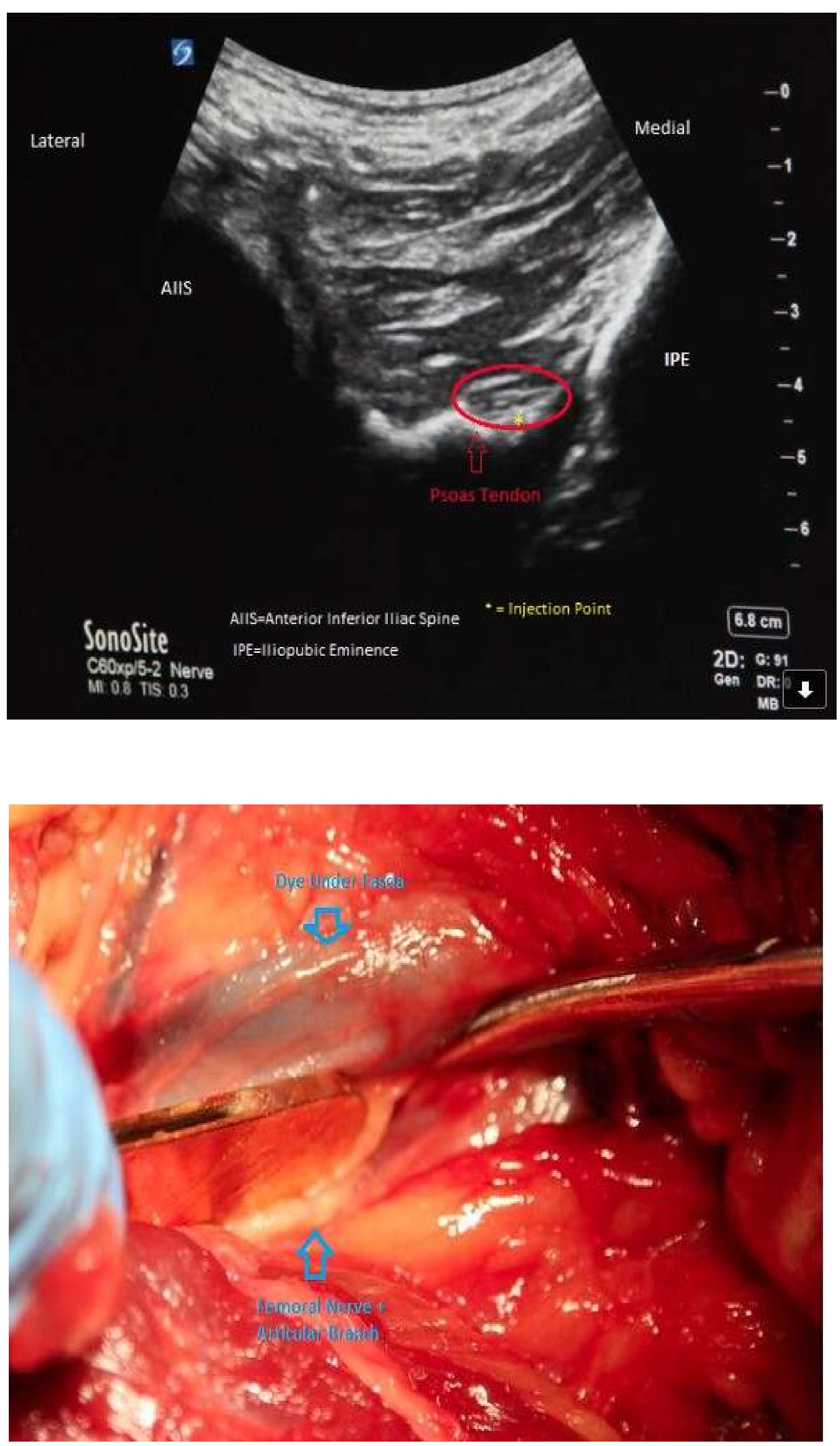

\section{Results}

Visualization of dye serves as proof-of-concept for this novel nerve block and provides an anatomical basis for its analgesic effects as described in recent literature. 